\title{
Evaluation of Dimensionless Flame Height Parameters to Account for Fuel Source Effects
}

\author{
Jarrod J. Alston \\ Arup Fire, New York, NY 10013, USA \\ Nicholas A. Dembsey \\ WPI Center for Firesafety Studies, Worcester, MA 01609, USA
}

\begin{abstract}
A key aspect of many flame height correlations is the use of a dimensionless scaling parameter. The $Q^{*}$ parameter, based on the total heat release rate of the fire, has been applied extensively to correlate the heights of turbulent diffusion flames, though comparison of correlations making use of $Q^{*}$ displays an apparent discrepancy. As a result it is not apparent that $Q^{*}$ is appropriate for flame heights. Delichatsios and Heskestad have determined that convective fraction of the fuel is an important parameter for flame heights and a correlation incorporating it would likely eliminate fuel dependence and result in a more refined prediction. The purpose of the present work was to develop a self-consistent set of data, where key characteristics are consistently defined, that can be used to parametrically explore the effect of fuel type, shape, and size. The data are then used to evaluate the ability of $Q^{*}$, and the correlating parameters of other researchers to successfully predict the variation in flame height due to fuel characteristics.
\end{abstract}

Key Words: flame height, dimensionless parameters, fuel type, heat release rate

\section{Introduction}

Obtaining a universal correlation for flame height or length of the visible portion of the fire plume is important in the field of fire protection engineering as such correlations are used in performance design analyses and engineering tools. Therefore, flame height predictions need to be as accurate as possible to assure that designs are being appropriately and accurately evaluated. Numerous flame height correlations that are currently used today are based on total heat release rate, making use of the $Q^{*}$ parameter developed by Zukoski, et al.[1]. It has been shown by several researchers that dimensionless flame heights correlate very well with $Q^{*}$, however the data often were limited to a single fuel or burner configuration. The functional form of the $Q^{*}$ correlations is as follows:

$$
\frac{z_{f}}{D}=\gamma Q^{* n} \cdots \text { where } Q^{*}=\frac{Q}{\rho_{\infty} c_{p} T_{\infty} g^{1 / 2} D^{5 / 2}}
$$

In Eq. 17 is the flame height, $D$ is the characteristic fuel dimension, $Q$ is the total heat release rate of the fire based on the theoretical or total heat of combustion multiplied by 
the fuel mass flow rate. The remaining variables, $\rho_{\infty}, c_{p}, T_{\infty}$, and $g$ are the ambient density, specific heat of the air, ambient temperature, and gravitational acceleration constant, respectively that define the ambient environment. The primary differences that exist between correlations are variations in the values of the constants $\gamma$ and $n$, and the range over which certain values of the coefficient and exponent are valid. Dembsey et al.[2] compared the various correlations and showed a lack of consistency over the range of research reviewed. Hasemi and Nshihata's[3] data for square burners was very similar to the data of Zukoski, et al.[1] for circular burners, indicating that there would be little shape effect. However, the data of Baum and McCaffrey[4] and of Cox and Chitty[5] would indicate that there is a significant effect when the shape of the burner is changed from circular to square, and of burner size. Delichatsios'[6] correlation for circular burners is similar to that of Zukoski, et al.[1] for $Q^{*}$ greater than 1.9, but tends to predict lower flame heights for $Q^{*}$ less than 1.9. Hasemi and Nishihata[3,7] and Sugawa et al.[8] showed that source geometry does not affect flame height for sufficiently large fires, $Q^{*}>1$, though noting that square burners tend to produce shorter flames due to extra mixing at the corners. There are further discrepancies among the correlations, including disagreement on power dependence. Adding to the uncertainty is that Delichatsios[6] and Heskestad[9,10] both found that the convective fraction, not accounted for in $Q^{*}$, was an important parameter in the analysis of fire plumes.

As a result, the use of $Q^{*}$ as the correlating variable for flame height is questionable. Zukoski[11] had stated as recently as 1994 that the appropriate scaling parameters for most of the features of a fire plume had not been established with confidence. Heskestad[12] evaluated several correlating variables, including $Q^{*[1]}$, Heskestad's $N$ number[9], Delichatsios' fire Froude number, $F_{f}[6]$; Steward's combustion number $N_{c o}$ [13], and correlating variables of Blake and McDonald[14,15], and Becker and Liang[16]. In the work, Heskestad determined that $N$ and $F r_{f}$ were virtually equivalent.

The present study, a continuation of work of Heskestad[12], and Quintiere and Grove[17] investigates the ability of the current non-dimensional parameters and correlations to effectively account for both fuel and shape effects on flame height. To do this, a series of experiments were conducted to provide a consistent data set for flame heights in the open that explores three global parameters that influence flame height, fuel convective fraction, $\chi_{\text {con }}$, burner shape (circular and square), and burner size $(0.17 \mathrm{~m}$ and $0.30 \mathrm{~m})$. As a result, the effect of each parameter could be explored individually, where characteristic dimensions such as source diameter and flame height are defined and measured on a consistent basis.

\section{Background and Theory \\ Correlating Variables}

The convective heat release rate is in theory a more accurate correlating variable than the total heat release rate because fire plumes are temperature driven, which is dependent on how much heat is radiated out of the plume. Quintiere and Grove[17] explained the dependence of flame height on fuel characteristics in terms of energy lost via radiation. Buoyancy caused by the temperature difference induces the velocity of the plume. As the plume gases travel in the vertical direction they entrain fresh air that is used for combustion. Entrainment is related to centerline velocity times a constant, such that the 
higher the centerline velocity (lower radiation loss) the higher the entrainment rate must be to make up for the upward moving plume gases. It has been contended by previous researchers that the flame height or height of the visible portion of the plume is directly related to the entrainment rate. The visible portion of the plume extends until all of the fuel has burned, thus the faster the plume entrains air the faster the fuel will be burnt, producing a lower flame height. Delichatsios described the effect of fuel type on flame height in terms of the formation of eddies. Buoyancy, which is related to convective flow, provides the energy for the formation of large eddies which engulf and mix fuel and ambient air; subsequently these eddies rapidly break up into smaller eddies by a turbulent cascade process. Delichatsios used the Froude number, which includes radiative fraction and efficiency of combustion (i.e. convective faction) to correlate flame heights[6].

Heskestad explains the inability of $Q^{*}$ to account for the effects of ambient conditions in terms of its development to correlate temperatures and velocities in far field non-reacting plumes and may not naturally extend to the combusting region of the plume[12]. The example Heskestad used to demonstrate the effectiveness of various correlating variables was the effect of variation of ambient temperature on flame height. Experimental data showed that at elevated temperatures, taller flames were seen and at depressed ambient temperatures shorter flames were seen, which was not accounted for in correlations using $Q^{*}$. He contends that $N$, found below, was developed specifically for the combustion zone, where the flame height model assumes that the flames extend to the height where sufficient air has been entrained for completion of the combustion reactions.

$$
N=\left[\frac{c_{p} T_{\infty}}{\left(H_{T} / r\right)}\right]^{3} Q^{* 2}
$$

The term $H_{T} / r$ is the theoretical or total heat of combustion divided by the stoichiometric air to fuel mass ratio. The amount of energy generated as a result of combustion is defined as the chemical energy, which is made up of a radiative and a convective component. The chemical heat of combustion, $H_{c h}$, is related to the total by the combustion efficiency, $\chi_{c h}=H_{c h} / H_{T}$. The convective fraction of the combustion efficiency is related to the chemical heat of combustion as $\chi_{c o n}=H_{c o n} / H_{c h}$. The radiative fraction is defined in a similar manner, where $\chi_{r a d}=H_{r a d} / H_{c h}$. Therefore, by definition, $\chi_{c o n}+\chi_{\text {rad }}=\chi_{c h}[18]$. The fuel convective fraction does not appear in the $N$ number itself, and thus combustion efficiency is not accounted for in $N$. Rather, Heskestad proposed that flame height was a function of both $N$ and the convective fraction such hat $z_{f} / D=f\left(N / \chi_{c o n}\right)$. However, the convective fraction was ultimately neglected as it was believed that it varies little over the range of interest, resulting in the following correlation that agreed well over a wide range of fires[10].

$$
z_{f} / D=-1.02+15.6 N^{1 / 5}
$$

Delichatsios[6] uses a fire Froude number as the correlating parameter for flame heights. The form of the Froude number is as follows:

$$
F r_{f}=\left[\frac{1}{\left(H_{T} /\left[(r+1) c_{p} T_{\infty}\right]\right)^{3 / 2}\left(\chi_{c h}-\chi_{r a d}\right)^{1 / 2}}\right] Q^{*}
$$


Like the $N$ number, $F r_{f}$ can be related to $Q^{*}$ with a simple scaling factor as seen in Eq. 4 . The functional form for determining the flame height is similar to that for $Q^{*}$ where the coefficient, $\gamma$, and exponent, $n$, vary depending on the value of $F r_{f}$.

Quintiere and Grove[17] have proposed unified theoretical correlations for flame height and entrainment of axisymmetric, line, and rectangular fire plumes based on the convective fraction of the heat release rate, thus explicitly incorporating combustion efficiency and plume buoyant strength. In the original expression, the heat release rate is based on the chemical heat of combustion. There appears some inconsistency in the development of the Quintiere and Grove correlation as the convective and radiative fractions were based on the chemical heat of combustion, yet the ratio of the heat of combustion to the stoichiometric ratio was based on the total heat of combustion. To compare with the correlations above it is necessary to convert Quintiere and Grove's formulation in terms of total heat release rate. The following general form of the correlation, with the previously mentioned modifications, has been developed based on relationships for rectangular shaped burners such that the limiting conditions for axisymmetric and line fires are satisfied.

$$
\begin{aligned}
& Q^{*}=0.00590 \frac{\Psi^{3 / 2}}{\left(\chi_{c h}-\chi_{\text {rad }}\right)}\left(\frac{z_{f}}{D}\right)^{1 / 2}\left(1+C_{1}\left(\frac{z_{f}}{D}\right)\right)^{m}\left(1+C_{1} a\left(\frac{z_{f}}{D}\right)\right)^{n} \\
& \text { where } \Psi=\frac{\left(\chi_{\text {ch }}-\chi_{\text {rad }}\right)\left(H_{T} / r\right)}{c_{p} T_{\infty}}
\end{aligned}
$$

The fire aspect ratio $a$ is defined as the short dimension divided by the long. The coefficient $C_{1}$, and the exponents $m$ and $n$ are respectively: axisymmetric $(0.357,1,1)$, rectangle $(0.398,1,1)$ and infinite line $(0.888,1,0)$. The fire characteristic dimension $\mathrm{D}$ is: axisymmetric (hydraulic diameter), rectangle (short side), and infinite line (line width). The correlation of Quintiere and Grove[17] agrees well with that of Heskestad[9] for $Q^{*}<1$. Quintiere and Grove propose that for the limits of aspect ratio (square vs. line), the power dependence of their correlation agrees well with that proposed by Zukoski[1]. However, over the range of interest in this study $\left.Q^{*} \sim 1\right)$, the power dependency deviates significantly from that of Zukoski and those observed of other researchers.

\section{Characteristic Dimensions}

Discrepancies in published data can potentially be explained in terms of various definitions and the measurement techniques used. For example, the definition of flame height differs from researcher to researcher. Zukoski, et al.[1] defined flame height intermittency as the horizontal height, $Z_{n}$, at which the flame is at or above for a given percentage of time. The $50 \%$ intermittent flame height $\left(Z_{0.50}\right)$ is the height at which the flame is at or above $50 \%$ of the time. The $100 \%$ intermittency height $\left(Z_{1.0}\right)$ is the height at which the flame is above $100 \%$ of the time and represents the minimum flame height. The $0 \%$ intermittency $\left(Z_{0.0}\right)$ is the maximum flame height and is the height at which the continuous flame is as tall as, but never taller than. Depending on the definition of flame height used, the resulting measurement can vary from 40 to $60 \%$ [1]. In addition, the measurement techniques can yield results that vary by as much as $15-20 \%[1,5]$. 
Also confounding the experimental data is the burner characteristic dimension or definition of diameter used by the researcher to correlate flame height. The most often used burner dimensions are diameter (for circles), hydraulic diameter, $D_{h}=4 A / P$, equivalent area diameter, $D_{e}=(4 A / \pi)^{1 / 2}$, or edge length. Where $\mathrm{A}$ is burner area and $\mathrm{P}$ is burner perimeter. For square sources the edge length and hydraulic diameter are equal, whereas they differ from the equivalent area diameter.

\section{Experimental Work}

The equipment and materials used for these tests include three different fuels, see Table 1 , and three different burners, Square $\left.Q_{h}=0.30 \mathrm{~m}, D_{e}=0.34 \mathrm{~m}\right)$, Circle $\left(D_{h}=0.30 \mathrm{~m}\right.$, $D_{e}=0.30 \mathrm{~m}$ ), and ISO [19](square shape: $D_{h}=0.17 \mathrm{~m}, D_{e}=0.19 \mathrm{~m}$ ). Two of the burners used (Circle, Square) had very similar construction in that the burners were constructed of 6 $\mathrm{mm}$ welded steel. The gas was fed in through the bottom of the burners through a welded Swagelok fitting. The gas stream then hits a baffle to disrupt the gas flow to result in low Reynolds number conditions and allow the fuel to diffuse evenly over the entire burner surfaces. A layer of $5 \mathrm{~cm}$ thick ceramic blanket (Kaowool) was clamped to the burner surfaces to act as a diffuser. The ISO burner, unlike the other burners, uses a layer of gravel and sand to act as a diffuser for the gaseous fuel.

The burners were located out in the open under a hood to collect products of combustion. The burner surface was approximately 0.5 meters off of the floor; a false floor was not placed around the burner. A screen was not placed around the burner to minimize drafts and air disturbances because flame height measurements were being made from video records and image quality was of high importance. In several experiments, disturbances notably affected flame heights. The results from such tests were neglected.

The gas cylinders (methane, propane, and propylene) were placed on a load cell with a resolution of $+/-0.1 \mathrm{~kg}$. Flow control of the fuel was achieved by passing the fuel through a regulator connected to the cylinder and then through a rotometer and needle valve. Flow conditions were continuously monitored for supply pressure and rotometer flow rate, which were first stabilized and then held constant throughout the course of each test. The test average heat release rate was determined by dividing the mass lost during the duration of the test by the test duration and by multiplying by the total heat of combustion of the fuel. The accuracy of the method is limited by the mass loss and time measurements. The corresponding average uncertainty in the heat release rate measurements, assuming the heats of combustion are known exactly, is approximately $+/-$ $6 \mathrm{~kW}$ expressed dimensionally, or $+/-0.2$ expressed non-dimensionally $\left(Q^{*}\right)$.

The set of experiments that were performed in this study consist of an array of 78 flame height tests. Roughly six to seven heat release rates were run for each fuel on each of the burners, ranging from $Q=6$ to $140 \mathrm{~kW}$ or $Q^{*}=0.2$ to 7 . Several data points with $Q^{*<1}$ were generated, and a number of data points where $Q^{*>1}$ were generated.

The flame heights of the steady diffusion flames were recorded using a video camera. Each experiment was run for a minimum of five minutes. The data was analyzed by taking three 150 consecutive frame sections and measuring the continuous peak heights. A number of computer programs were employed to assist in measuring the flame heights. The first step was to capture the three segments from the VHS video recordings to digital 
AVI ( $640 \times 480$ resolution) format; this was done by using a video capture card. The videos were then edited in Adobe Premiere to generate a single 450 frame Quicktime format movie. The flame heights from the Quicktime movies were then measured using an educational program called VideoPoint that allowed coordinate axes to be set and the length scales to be calibrated using known distances from each video. The distance used for length scale calibration within the program was taken from the ruler, that had $5 \mathrm{~cm}$ markings and was located adjacent to the burner during all tests. The program tracks flame height by allowing the user to pick the point of the flame tip in each video frame, automatically recording flame height with respect to time. The accuracy of the method, dependent on the resolution of the film and the overall flame height (i.e. accuracy is improved for smaller flame heights), is on average $+/-5 \mathrm{~cm}$, or $+/-0.2$ expressed in nondimensional terms $\left(Z_{n} / D\right)$. The accuracy is a composite of the uncertainty in the instantaneous measurement and in the length-scale calibration. The 450 heights were then compiled and the data reduced to determine the intermittent flame heights of the fires calculated.

Table 1: Key parameters of the three fuels used in this study.

\begin{tabular}{|c|c|c|c|c|c|}
\hline \multirow{2}{*}{ Fuel } & \multicolumn{3}{|c|}{ Heats of Combustion [MJ/kg]/ Fractions [] } & \multirow{2}{*}{$\begin{array}{c}\text { Stoich. } \\
\text { Air/Fuel, } \mathrm{r}\end{array}$} \\
\cline { 2 - 5 } & Total & Chemical & Convective & Radiative & Air/ \\
\hline Methane (98\% pure) & 50.1 & $49.6 / 0.99$ & $42.6 / 0.85$ & $7.0 / 0.14$ & 17.16 \\
\hline Propane (pure) & 46.0 & $43.7 / 0.95$ & $31.2 / 0.68$ & $12.5 / 0.27$ & 15.60 \\
\hline Propylene & 46.4 & $40.5 / 0.87$ & $25.6 / 0.55$ & $14.9 / 0.32$ & 14.70 \\
\hline
\end{tabular}

Results and Discussion

Fuel Dependence - Radiative Fraction

One of the primary goals of this study was to evaluate the effect of the fuel type or convective fraction on the resulting flame height. As mentioned previously, it is believed that flame height is inversely related to the near field entrainment rate of the fire plume, which is in turn driven by the convective heat release rate of the fire itself. It would then be expected that for equivalent total heat release rates for the three fuels used in this study, that the flames from methane $\left.\chi_{\text {con }}=0.85\right)$ fires would be shorter than those for propane $\left(\chi_{\text {con }}=0.68\right)$ fires, which would in turn be smaller than those for propylene $\left(\chi_{\text {con }}\right.$ $=0.55)$ fires. The data from the present study for similarly sized axisymmetric sources (Circle and Square burners) are plotted according to fuel type in Fig. 1. Square burners will be considered axisymmetric fuel sources in the near field above the burner surface. The measured flame heights for both Circle and Square burners indicate little variation and are most often within an uncertainty of $+/-3 \mathrm{~cm}$ or $+/-0.1$ in non-dimensional terms $\left(Z_{n} / D\right)$ which falls within the non-dimensional uncertainty bands associated with the data and the measurement techniques, making it impossible to say with confidence that the differentiation in behavior is physical or a result of measurement uncertainty. Thus, it can be assumed that the flame heights of both circles and squares are very nearly similar, especially in the near field well above the burner surface where the flame retains no "memory" of the fuel source[5]. All data are plotted against $Q^{*}$ based on total heat release rate as a bench mark in order to display how fuel parameters effect the flame height. 


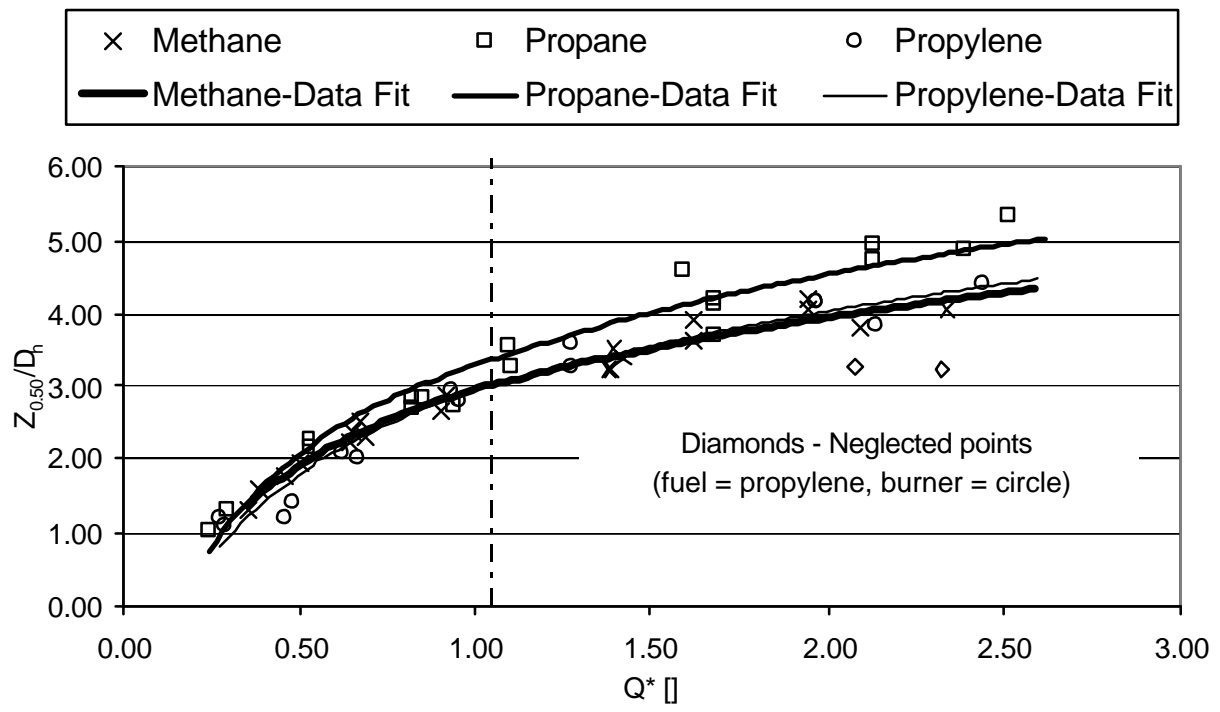

Fig. 1: Effect of fuel characteristics (convective fraction) on flame height from axisymmetric $(0.3 \mathrm{~m}$ square and $0.3 \mathrm{~m}$ circle burners) fuel sources. The vertical line represents the point at which the deviation between flame heights of different fuels exceeds twice the uncertainty in the flame height measurements.

What is evident from Fig. 1 is that a simple linear relationship between flame height and convective heat release does not exist. There is some scatter and uncertainty evident in the data due to the limitations associated with "real" scale fires and the inability to fully control ambient conditions. Trendlines have been added to effectively smooth the data and emphasize any relationships that may exist. The uncertainty associated with each flame height measurement is low, therefore the trendlines are believed to reflect actual behavior evidenced in the data and not simple scatter or uncertainty for $Q^{*}$ greater than approximately 1.05 . For fires below $Q^{*}=1.05$, the variation in flame height is of the same order of magnitude as the measurement uncertainty. Selected apparent outliers, where flames were visibly disturbed by the ambient environment, were neglected in the generation of the best-fit curves as it was judged that outliers in such a moderately sized data set could skew the data and imply non-physical trends or relationships.

Based on the data it is evident that the relationship is not a simple scaling in flame height based on the convective fraction. Simply multiplying the heat release rate by he convective fraction as recommended by Anderson[20], does not agree with the trend seen in comparing the methane flames to the propane flames to the propylene flames. Such a trend contradicts the theory proposed earlier that the flame height is related $\mathfrak{b}$ the entrainment rate in that a flame that radiates more of it energy away will require a larger flame zone in order for the fuel to be totally consumed. Thus, the data support Zukoski's[11] statement, and later Heskestad's[12] argument that $Q^{*}$ is not the appropriate correlating variable for flame height. 

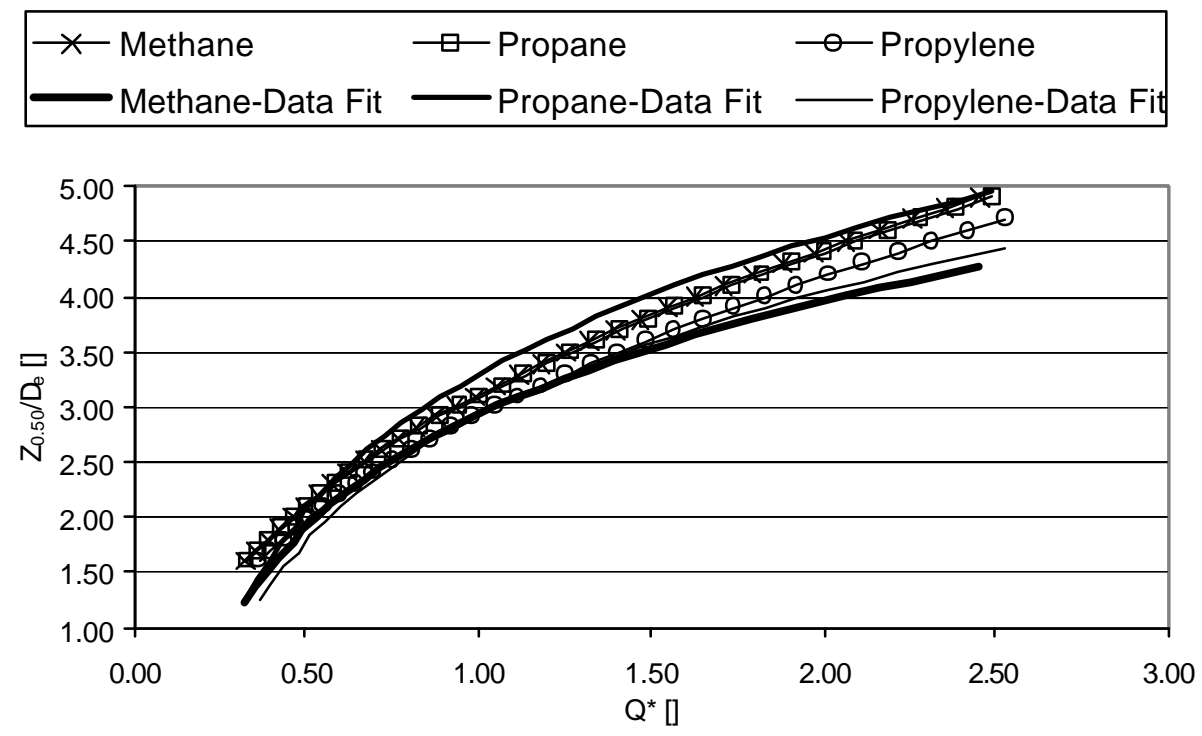

Fig. 2: Flame height versus non-dimensional total heat release rate calculated using the correlation of Heskestad[9] (points with lines) plotted with data best fit lines.

Next, the correlations of Heskestad[9], Quintiere and Grove[17], and Delichatsios[6] were evaluated for their ability to model the fuel dependent behavior seen in the present study. Given the differences in flame height due to fuel type, it was necessary to get a more definitive look at how each correlation accounts for the variation relative to a common variable. To do this, each correlation has been plotted versus $Q^{*}$.

Heskestad's correlation[9], pictured in Fig. 2, differentiates propylene flame heights from the other two fuels, but since convective fraction was neglected in the development of the correlation it is not surpris ing that the correlation does not predict taller flames for propane relative to methane fires. Predicted non-dimensional flame heights using Heskestad's correlation fall within the data trend lines indicating that the predicted flame heights are in general agreement with the measured data. The correlation, however, under-predicts the variation in flame height observed between propylene and propane. For $Q^{*}>1$, the dependence of $Z_{0.50} / D \propto Q^{2 / 5}$ in the correlation is consistent with what was observed in the tests.

Quintiere and Grove's correlation[17], pictured in Fig. 3 is unable of differentiating between propylene and propane, predicting similar non-dimensional flame heights for the two fuels. The correlation, however, is able to predict the fuel effects of methane on flame height, relative to the other two fuels. The correlation under-predicts flame heights relative to the best-fit lines from the data for $0.5<Q^{*}<2$. There also appears to be a discrepancy between the power dependence indicated by the trendlines relative to the correlation predictions within the same range of $Q^{*}$, which is consistent with the previous observation regarding the disagreement of power dependence for "moderate" values of $Q^{*}$. The results using the correlation of Quintiere and Grove indicate flame height is inversely related to convective fraction and the ratio of the total heat of combustion divided by the stoichiometric ratio, both raised to a power. For the axisymmetric case, 

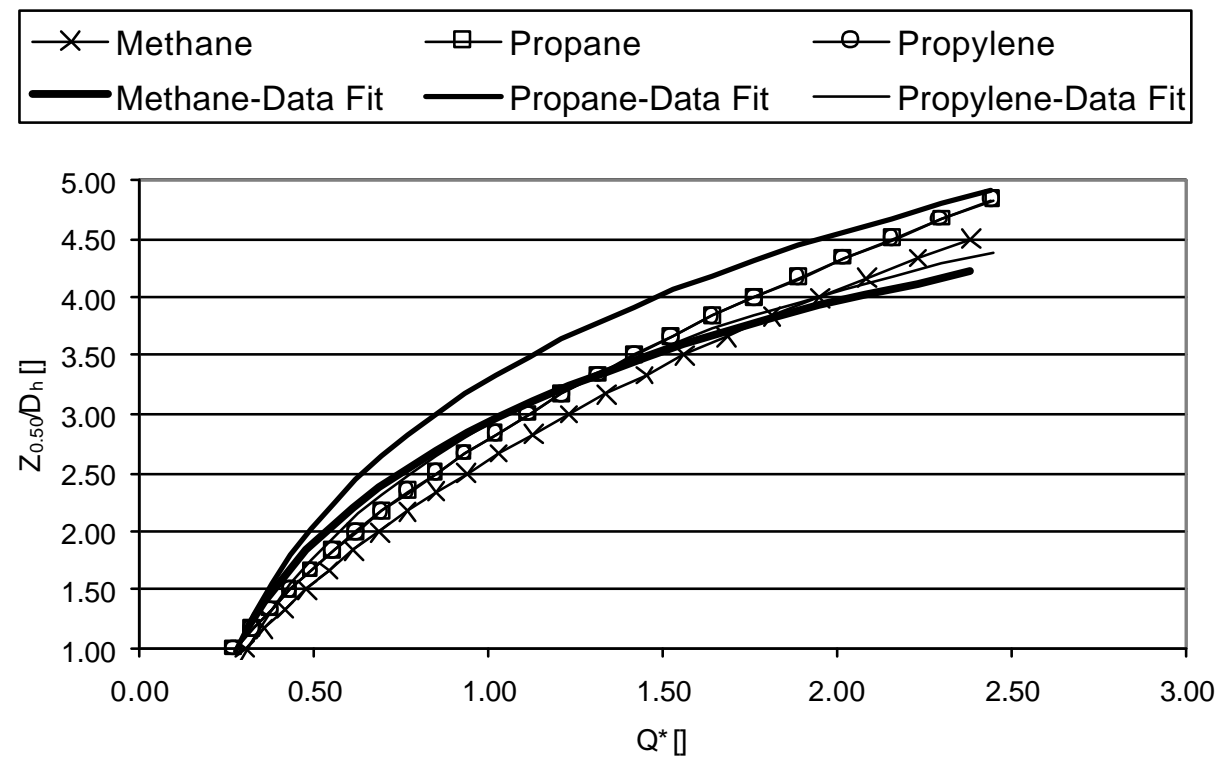

Fig. 3: Flame height versus non-dimensional total heat release rate calculated using the correlation of Quintiere and Grove[17] (points with lines) plotted with data best fit lines.

the power dependencies for the various factors are roughly equivalent to the correlation of Delichatsios. Regardless of any success of the Quintiere and Grove correlation in accounting for fuel effects, the use of the correlation is cumbersome since the mean flame height cannot be solved for explicitly.

Delichatsios' $[6]$ correlation is pictured in Fig. 4, and shows that 1ke Quintiere and Grove's correlation[17], it is capable of discriminating the differences in flame height between methane and propane fires. However, like the predictions using Quintiere and Grove's correlation, propylene flames are predicted to be the same height as propane flames, which is counter to what was seen in the experiments. Delichatsios' correlation tends to under-predict the observed flame heights below approximately $Q^{*}<1.7$ and overpredicts flame heights at $Q^{*}>1.7$. Furthermore, the power dependence for small flames (i..e $Q^{*}<1$ ) is not in agreement with the trends seen in the data.

Although the three correlations reviewed are similar in form, there is a lack of agreement among them as to the effect of the fuel type. From Fig. 2 through Fig. 4, it appears that convective fraction and the quotient of the total heat of combustion divided by the stoichiometric air to fuel ratio are important correlating parameters. However, the exact dependence of non-dimensional flame height on either parameter still remains unclear.

Fuel Dependence - Burner Size

As mentioned previously, comparison of the data of Baum and McCaffrey[4] to that of Cox and Chitty[5] indicate an effect of burner size on non-dimensional flame height. 

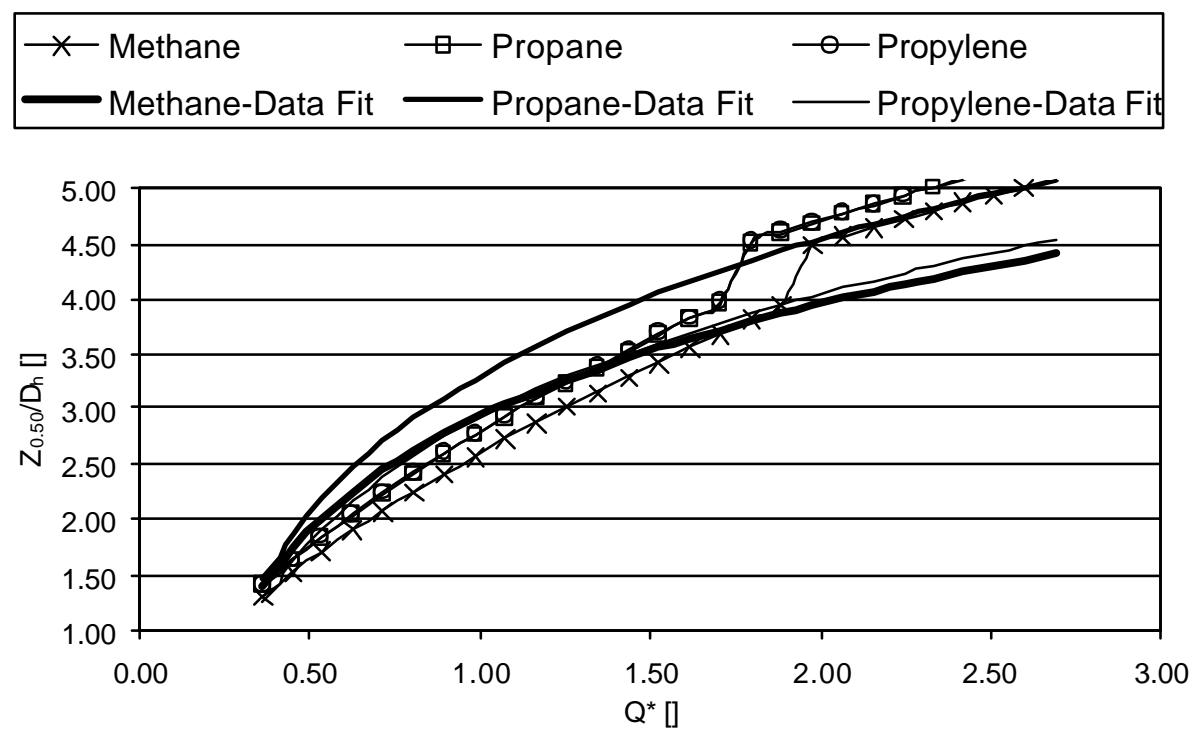

Fig. 4: Flame height versus non-dimensional total heat release rate calculated using the correlation of Delichatsios[6] (points with lines) plotted with data best fit lines.

This trend, however, is not observed in the data of Zukoski, et al.[1] It was therefore of interest to determine if there was an effect on flame height as a result of the burner size.

Although the burners are of dissimilar construction, the tests on the Square and the ISO burners provide a means of checking this hypothesis. The non-dimensional flame heights on the ISO and Square burners fall well within the non-dimensional uncertainty bands. However, additional tests on burners of a larger range of sizes (i.e. $D_{h}<0.17 \mathrm{~m}$ and $D_{h}>$ $0.30 \mathrm{~m}$ ) would need to be completed to verify this observation.

\section{Conclusions}

The currently available flame height correlations are typically believed to be adequate for predicting flame heights within an uncertainty of approximately 15 to $20 \%$. However, the most often used correlations make use of the $Q^{*}$ parameter, which neglects fuel properties. For most engineering calculations, use of $Q^{*}$ is acceptable since fuel characteristics, including type and size, often need to be assumed. However, more refined predictions can be made by using correlations, such as that of Quintiere and Grove[17], that incorporate fuel properties such as convective fraction. The current work develops a self-consistent set of data using new techniques for measuring visible flame heights. The attempt is not to define the mechanisms that cause the discrepancies seen in flame heights, but rather to explore the ability of current correlations to predict observed behavior. Conclusions that can be drawn from the current work include:

There is a flame height fuel dependence as evidenced by the differing trends seen in the plots of the flame heights for the three different fuels used in this study versus $Q^{*}$ based on total heat release rate. However, the fuel dependence is not a simple scaling relationship with the assumed constant convective fraction as evidenced by the results, 
which did not show a clear trend in terms of increased flame height with lower convective heat release rate.

As a result of the trend observed, it was desirable to evaluate if the current flame height correlations and correlating variables were able to predict the observed fuel dependent behavior. It was found that simply reducing the heat release rate in the $Q^{*}$ calculation by the convective fraction was insufficient in modeling the flame height behavior. Heskestad's $N$ number[9], the semi-empirical formulation by Quintiere and Grove[17], and Delichatsios' [6] correlation were explored. It was found that the $N$ number was able to capture the trend of lower observed flame heights as seen for propylene, but was unable to discriminate between the methane and propane flames. This was due to the $\left(H_{T} / r\right)^{3}$ factor in the denominator of the $N$ number. It was found that Quintiere and Grove's derivation was capable of discriminating between the flame heights of propane and methane fires. However, the correlation showed no variation between propylene and propane flames. The prediction of methane flames being shorter than propane flames is consistent with experimental observations. Thus, the present work is an extension of the original work by Quintiere and Grove by applying the correlation to a wider range of experimental data, to evaluate the assumptions made in its development. The approximate relationship between non-dimensional flame height and the relevant parameters is:

$$
Z_{0.50} / D \propto Q^{2 / 5}\left(\chi_{c h}-\chi_{\text {rad }}\right)^{-1 / 5}\left(H_{T} / r\right)^{-3 / 5}
$$

The relationship is consistent with the $N$ number correlation and with the Froude number[6], $F r_{f}$, in terms of the power dependence of both $H_{T} / r$ and convective fraction. This would give support to use of some form of $\mathrm{Fr}_{f}$ as an appropriate correlating variable for flame height, except that the predicted propylene flame heights are too high for values of $Q^{*}>1.7$ and too low for values of $Q^{*}<1.7$.

The present study also explored the effect of burner shape on flame height. It was found that there was no discernible difference in measured flame heights on circular and square burners for the flame heights investigated. Two different sized square burners were used in testing. The larger burner had an edge length of 0.30 meters while the smaller (ISO) burner had an edge length of 0.17 meters. There appeared to be no effect of burner size on non-dimensional flame height.

\section{Acknowledgments}

The support received from the SFPE Educational and Scientific Foundation is greatly appreciated. The help of all those who assisted in the experiments is appreciated, as is the preliminary work conducted by Scott Anderson.

\section{References}

1 Zukoski, E., T. Kubota, and B. Cetegen, "Entrainment in Fire Plumes," Fire Safety Journal, 3: 107-122 (1981). 
2 Dembsey, N.A., Pagni, P.J., and Williamson, R.B., "Compartment Fire Near Field Entrainment Measurements", Fire Safety Journal, 24: 383-419 (1995).

3 Hasemi, Y. and M. Nishihata, "Fuel Shape effect on the Deterministic Properties of Turbulent Diffusion Flames", Fire Safety Science - Proceedings of the Second International Symposium, International Association for Fire Safety Science, Hemisphere Publishing Corporation, New York, USA, 1989, pp. 275-284.

4 Baum, H. and B. McCaffrey, "Fire Induced Flow Field-Theory and Experiment," Fire Safety Science - Proceedings of the Second International Symposium, International Association for Fire Safety Science, Hemisphere Publishing Corporation, New York, USA, 1989, pp. 129-148.

5 Cox, G. and R. Chitty, "Some Source-Dependent Effects of Unbounded Fires," Combustion and Flame, 60: 219-232 (1985).

6 Delichatsios, M.A., "Procedure for Calculating Air Entrainment into Turbulent Pool and Jet Fires", Journal of Fire Protection Engineering, 2: 93-98 (1990).

7 Hasemi, Y. and M, Nishihata, "Deterministic Properties of Turbulent Diffusion Flame from Low Q* Fires," Fire Science \& Technology, 7: 27-34 (1987).

8 Sugawa, O., H. Satoh, and Y. Oka, "Flame Height From Rectangular Fire Sources Considering Mixing Factor," Fire Safety Science - Proceedings of the Third International Symposium, International Association for Fire Safety Science, London, UK, 1991, pp. 435-444.

9 Heskestad, G., "Peak gas Velocities and Flame Heights of Buoyancy-Controlled Turbulent Diffusion Flames", 18th Symposium International on Combustion, The Combustion Institute, Pittsburgh, PA, USA, 1981, pp. 951-959.

10 Heskestad, G., "Luminous Height of Turbulent Diffusion Flames," Fire Safety Journal, 5: 103-108 (1983).

11 Zukoski, E. "Mass Flux in Fire Plumes," Fire Safety Science - Proceedings of the Fourth International Symposium, International Association for Fire Safety Science, London, UK, 1994, pp. 137-147.

12 Heskestad, G., "On Q* and the Dynamics of Turbulent Diffusion Flames," Fire Safety Journal, 30: 215-227 (1998).

13 Steward, F., "Prediction of the Height of Turbulent Diffusion Buoyant Flames," Combustion Science and Technology, 2: 203-212 (1970).

14 Blake, T. and M. McDonald, "An Examination of Flame Length Data from Vertical Turbulent Diffusion Flames," Combustion and Flame, 94: 426-432 (1993).

15 Blake, T. and M. McDonald, "Similitude and the Interpretation of Turbulent Diffusion Flames," Combustion and Flame, 101: 175-184 (1995).

16 Becker, H. and D. Liang, "Visible Length of Vertical Free Turbulent Diffusion Flames," Combustion and Flame, 32: 115-137 (1978).

17 Quintiere, J. and B. Grove, "Correlations for Fire Plumes", National Institute for Standards and Technology, NIST-GCR-98-744, Gaithersburg, MD, USA, 1998, 12 p. 18 Tewarson, A. "Generation of Heat and Chemical Compounds in Fires," The SFPE Handbook of Fire Protection Engineering ( $\left.2^{\text {nd }} e d\right)$, NFPA, Quincy, MA, 02269, 1995, Ch. 3-4.

19 "ISO 9705 Full Scale Room Test for Surface Products", International Organization for Standardization, Geneva, Switzerland, 1993.

20 Anderson, S.K., and N.A. Dembsey, " Qualitative Study of the Effect of a Compartment Enclosure on Fire Plume Entrainment”, WPI Master's Thesis, Worcester, MA, USA, 1998. 